\title{
Bootstrapped Text-level Named Entity Recognition for Literature
}

\author{
Julian Brooke Timothy Baldwin \\ Computing and Information Systems \\ The University of Melbourne \\ jabrooke@unimelb.edu.au \\ tb@ldwin. net
}

\author{
Adam Hammond \\ English and Comparative Literature \\ San Diego State University \\ ahammond@mail.sdsu.edu
}

\begin{abstract}
We present a named entity recognition (NER) system for tagging fiction: LitNER. Relative to more traditional approaches, LitNER has two important properties: (1) it makes no use of handtagged data or gazetteers, instead it bootstraps a model from term clusters; and (2) it leverages multiple instances of the same name in a text. Our experiments show it to substantially outperform off-the-shelf supervised NER systems.
\end{abstract}

\section{Introduction}

Much of the work on applying NLP to the analysis of literature has focused on literary figures/characters in the text, e.g. in the context of social network analysis (Elson et al., 2010; Agarwal et al., 2013; Ardanuy and Sporleder, 2015) or analysis of characterization (Bamman et al., 2014). Named entity recognition (NER) of person names is generally the first step in identifying characters; locations are also a prevalent NE type, and can be useful when tracking different plot threads (Wallace, 2012), or trends in the settings of fiction.

There are not, to our knowledge, any NER systems that are specifically targeted at literature, and most related work has used Stanford CoreNLP as an off-the-shelf solution (Bamman et al., 2014; Vala et al., 2015). In this paper, we show that it is possible to take advantage of the properties of fiction texts, in particular the repetition of names, to build a high-performing 3-class NER system which distinguishes people and locations from other capitalized words and phrases. Notably, we do this without any hand-labelled data whatsoever, bootstrapping a text-level context classifier from a low-dimensional Brown clustering of the Project Gutenberg corpus.

\section{Related work}

The standard approach to NER is to treat it as a supervised sequential classification problem, typically using conditional random fields or similar models, based on local context features as well as properties of the token itself. Relevant to the present work is the fact that, despite there being some work on enforcing tag consistency across multiple instances of the same token (Finkel et al., 2005) and the use of non-local features (Ratinov and Roth, 2009) to improve supervised sequential models, the consensus seems to be that this nonlocal information has a relatively modest effect on performance in standard datasets, and as a result off-the-shelf NER systems in practice treat each sentence as a separate document, with multiple instances of the same token in different sentences viewed as entirely independent classification problems. We also note that although supervised NER is the norm, there is a smaller body of work in semi-supervised and unsupervised approaches to NER and semantic lexicon induction, for instance pattern bootstrapping (Nadeau et al., 2006; Thelen and Riloff, 2002; McIntosh et al., 2011) as well as generative approaches (Elsner et al., 2009).

In the context of literature, the most closely related task is character identification (Vala et al., 2015), which is itself an intermediate task for character speech identification (He et al., 2013), analysis of characterization (Bamman et al., 2014), and analysis of social networks (Elson et al., 2010; Agarwal et al., 2013; Ardanuy and Sporleder, 2015). In addition to NER, character identifica- 
tion also involves clustering multiple aliases of the same character, and discarding person names that don't correspond to characters. Vala et al. (2015) identify some of the failures of off-the-shelf NER with regards to character identification, and attempt to fix them; their efforts are focused, however, on characters that are referred to by description rather than names or aliases.

\section{Method}

\subsection{Corpus preparation and segmentation}

The corpus we use for building and testing our NER system is the 2010 image of the (US) Project Gutenberg corpus, ${ }^{1}$ a reasonably comprehensive collection of out-of-copyright English literary texts, to our knowledge the largest that is publicly available in a machine-readable, full-text format. We access the texts via the GutenTag tool (Brooke et al., 2015), which allows both filtering of texts by genre as well as within-text filtering to remove Project Gutenberg copyright information, front and back matter (e.g. table of contents), and headers. We focus here only on fiction texts (i.e. novels and short stories); other kinds of literature (e.g. plays) are rare in the corpus and have very different properties in terms of the distribution of names. The final corpus size is 10844 texts.

GutenTag also provides an initial segmentation of tokens into potential names, using a simple rule-based system which segments contiguous capitalized words, potentially with common intervening function words like of as well as leading the (e.g. the King of Westeros). It largely (but not entirely) overcomes the problem of sentenceinitial capitalization in English by generalizing over an entire text; as long as a capitalized word or phrase appears in a non-sentence initial position at least once in a text, it will be tagged in the sentence-initial position as well. To improve precision, the name tagger in the version of GutenTag used for this paper (0.1.3) has lower bounds on token count (at least 10) and an upper bound on the length of names (no longer than 3 words). For this work, however, we remove those restrictions to maximize recall. Though not our primary concern, we return to evaluate the quality of the initial segmentation in Section 5.

\footnotetext{
${ }^{1}$ http://www.gutenberg.org
}

\subsection{Brown clustering}

The next step is to induce Brown clusters (Brown et al., 1992) over the pre-segmented corpus (including potential names), using the tool of Liang (2005). Briefly, Brown clusters are formed using an agglomerative hierarchical cluster of terms based on their immediate context, placing terms into categories to maximize the probability of consecutive terms over the entire corpus. Note that using information from Brown clusters is a well established technique in NER, but more typically as features within a supervised framework (Miller et al., 2004; Liang, 2005; Ritter et al., 2011); we are unaware of any work using them directly as a source of bootstrapped training examples. We used default settings except for the number of clusters $(c)$ : 50 . The rationale for such a small cluster size - the default is 1000 , and NER systems which use Brown clusters as features do better with even more (Derczynski et al., 2015) - is that we want to have clusters that correspond to major noun categories (e.g. PERSON and LOCATION), which we consider the next most fundamental division beyond part-of-speech; 50 was selected because it is roughly comparable to the size of the Penn Treebank tagset (Marcus et al., 1993). We did not tune this number, except to observe that larger numbers (e.g. 100 or 200) resulted in increasingly fragmented clusters for our entities of interest.

To automatically extract a seed list of people and locations, we ranked the clusters by the total (token) count of names (as identified by GutenTag), and took the first cluster to be PERSON, and the second to be LOCATION; all other clusters are considered OTHER, our third, catchall category. Alternatively, we could have set $c$ higher and manually grouped the clusters based on the common words in the clusters, adding a thin layer of supervision to the process; with a low $c$, however, this was unnecessary since the composition and ranking of the clusters conformed exactly to our expectations. The top-5 clusters by token count of names are given in Table $1 .^{2}$ Note the presence of the multiword name New York in the second cluster, as a result of the segmentation.

The most common words in the first two clusters are mostly what we would expect, though there is a bit of noise, e.g. Him included as a place. The other clusters are messier, but still in-

\footnotetext{
${ }^{2}$ Note that each cluster generally includes large numbers of non-names, which we ignore.
} 


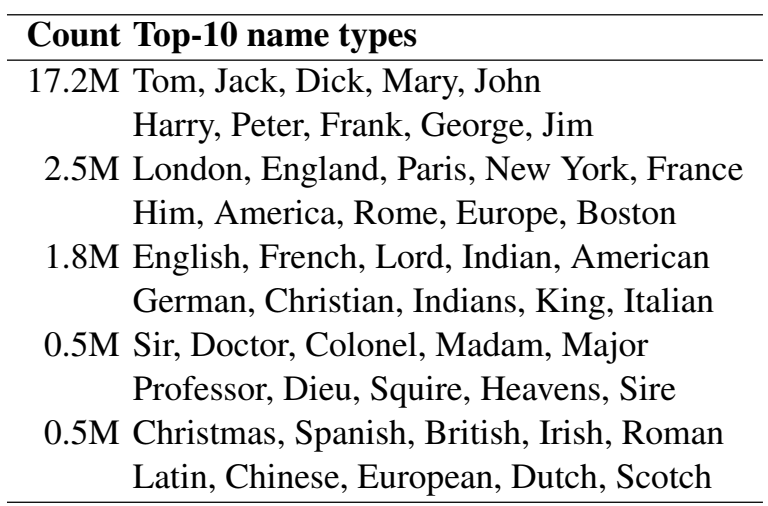

Table 1: Top-5 Brown clusters derived from PG corpus, by token count of names

terpretable: e.g. Cluster 4 is a collection of terms of address. Note that although we do not consider an term like Doctor to be a person name, Doctor Smith or the Doctor would be; in many literary contexts characters are referred to by an alias, and failure to deal properly with these situations is a significant problem with off-the-shelf NER systems in literature (Vala et al., 2015). In any case, Brown clustering works fairly well for common names, but for rarer ones, the clustering is haphazard. Fiction, though, has many rare names and locations, since authors will often invent them. Another problem with Brown clustering is that ignores possible sense distinctions: for instance, Florence is both a city and a person name. To avoid confusion, authors will generally preserve one-sense-per-document, but this is not true at the corpus level.

\subsection{Text-level context classifier}

The central element of our NER system is a textlevel classifier of names based on context. By text-level, we mean that it assumes one-sense-perdocument, classifying a name for an entire document, based on all instances of the name in the document (Gale et al., 1992). It is trained on the (text-level) "instances" of relatively common names (appearing more than 100 times in the corpus) from the $3 \mathrm{NE}$ label types derived based on the Brown clustering. That is, to build a training set, we pass through the corpus and each time we come across a common name in a particular document, we build a feature vector corresponding to all the contexts in that document, with the label taken from the clustering. Our rationale here is that the challenging part of NER in literature is names that appear only in one text; by limiting our context for common words to a single text, we simulate the task for rarer words. Mary is a common name, and may be a major character in one text, but a minor one in another; hence, we build a classifier that deals with both context-rich and context-poor situations. The noisy training set thus constructed has about 1 million examples.

Our feature set consists of filtered word features in a 2-word window $\left(w_{-2} w_{-1} w_{0} w_{+1} w_{+2}\right)$ around the token occurrences $w_{0}$ of a target type in a given text, made up of position-indexed unigrams $\left(w_{-2}, w_{-1}, w_{+1}\right.$ and $\left.w_{+2}\right)$ and bigrams $\left(w_{-2} w_{-1}, w_{+1} w_{+2}\right.$ and $\left.w_{-1} w_{+1}\right)$, excluding unigrams when a subsuming bigram feature matched (e.g. if we match trust in, we do not add trust and in). For this we used the name-segmented corpus, and when one of the words in the context was also a name, we take the category from the Brown clustering as the word (so $w_{2}$ for London in from London to New York is LOCATION, not New). Across multiple tokens of the same type, we count the same context only once, creating a binary feature vector which was normalized by dividing by the count of all non-zero entries once all contexts were collected. To be included as features, the $n$-grams had to occur with $\geq 10$ different $w_{0}$ target word types. Note that given our bootstrapping setup, the word type itself cannot be used directly as a feature.

For classification, we use logistic regression from scikit-learn (Pedregosa et al., 2011) trained with SGD using $\mathrm{L} 2$ regularization $(C=1){ }^{3}$ The only non-standard setting that we use is the "balanced" option, which weights classes by the inverse of their count in the training set, countering the preference for the majority class; we do this because our bootstrapped distribution is an unreliable reflection of the true distribution, and also because it makes it a fairer comparison to off-theshelf models with no access to this distribution.

\subsection{Improved phrase classification}

Relative to (true) supervised models, our bootstrapped model suffers from being able to use only context, and not the identity of the name itself. In the case of names which are phrases, this is troubling because there are many generalizations to be made; for instance names ending with City are locations. Our final model addresses this failing somewhat by using more information from our

\footnotetext{
${ }^{3}$ Using cross-validation over the training data, we tested other solvers, L1 regularization, and settings of the $C$ parameter, but saw no appreciable improvement in performance.
} 
Brown clustering: from each of the initial and final words across all names, we extract a set of words $W_{s}$ that appear at least ten times in position $s \in S, S=\{$ initial, final $\}$ across all phrases. Let $c(w, t, s)$ be the the number of times a word $w \in W_{s}$ appears in the corpus at position $s$ in phrases which were Brown clustered into the entity type $t \in T$, and $p(t \mid r)$ be the original probability of phrase $r$ being type $t$ as determined by the logistic regression classifier. For our two homogenous entity types (PERSON and LOCATION), we calculate a new score $p^{\prime}$ :

$$
\begin{array}{r}
p^{\prime}(t \mid r)=p(t \mid r)+\sum_{s \in S}\left(\frac{c\left(r_{s}, t, s\right)}{\sum_{t^{\prime} \in T} c\left(r_{s}, t^{\prime}, s\right)}\right. \\
\left.-\frac{\sum_{w^{\prime} \in W_{s}} \frac{c\left(w^{\prime}, t, s\right)}{\sum_{t^{\prime} \in T} c\left(w^{\prime}, t^{\prime}, s\right)}}{\left|W_{s}\right|}\right)
\end{array}
$$

The first term in the outermost summation in Equation 1 is the proportion of occurrences of the given expression in position $s$ which correspond to type $t$. To avoid applying too much weight to the homogeneous classes, the second term in the summation subtracts the average number of occurrences in the given position for all words in $W_{s}$. As such, the total effect on the score can be negative. Note that if $r_{s} \notin W_{s}$, no modification is made, and for the OTHER type $p^{\prime}(t \mid r)=p(t \mid r)$. Once we have calculated $p^{\prime}(t \mid r)$ for each class, we choose the $t$ with the highest $p^{\prime}(t \mid r)$.

\section{Evaluation}

Our interest is in a general NER system for literature. Though there are a few novels which have been tagged for characters (Vala et al., 2015), we wanted to test our system relative to a much wider range of fiction. To this end, we randomly sampled texts, sentences, and then names within those sentences from our name-segmented Project Gutenberg corpus to produce a set of 1000 examples. These were tagged by a single annotator, an English native speaker with a $\mathrm{PhD}$ in English Literature. The annotator was presented with the sentence and the pre-segmented name of interest, and asked (via written instructions) to categorize the indicated name into PERSON, LOCATION, OTHER, UNCERTAIN due to ambiguity, or segmentation error. We ran a separate two-annotator agreement study over 200 examples which yielded a Cohen's Kappa of 0.84, suggesting high enough reliability that a single annotator was sufficient. The class

\begin{tabular}{lcc}
\hline System & Acc & $\mathcal{F}_{\mathbf{M}}$ \\
\hline All PERSON baseline & .696 & - \\
OpenNLP & .435 & .572 \\
LingPipe & .528 & .536 \\
Stanford CoreNLP & .786 & .751 \\
Brown clusters & .803 & .672 \\
\hline LitNER sentence +phrase & .757 & .671 \\
LitNER text - phrase & .855 & .771 \\
LitNER text + phrase & $\mathbf{. 8 7 1}$ & $\mathbf{. 7 9 2}$ \\
\hline
\end{tabular}

Table 2: Performance of NER systems

distribution for the main annotation was $66.9 \%$ PERSON, $10.2 \%$ LOCATION, $19.0 \%$ OTHER, $2.4 \%$ UNCERTAIN, and $1.5 \%$ segmentation error. For the main evaluation, we excluded both UNCERTAIN examples and segmentation errors, but had our annotator provide correct segmentation for the 15 segmentation errors and carried out a separate comparison on these.

We compare our system to a selection of publicly available, off-the-shelf NER systems: OpenNLP, ${ }^{4}$ LingPipe, ${ }^{5}$ and Stanford CorenLP (Finkel et al., 2005), as well as the initial Brown clustering. OpenNLP allowed us to classify only PERSON and LOCATION, but for Stanford CoreNLP and LingPipe we used the existing 3-entity systems, with the ORGANIZATION tag collapsed into OTHER (as it was in our guidelines; instances of ORGANIZATION are rare in literature). Since the exact segmentation guidelines likely varied across these systems-in particular, we found that Stanford CorenLP often left off the title in names such as Mr. Smithand we didn't want to focus on these issues, we did not require exact matches of our name segmentation; instead, we consider the entire name as PERSON or LOCATION if any of the tokens were tagged as such (names with both tags were considered OTHER). For our system (LitNER), we test a version where only the immediate sentence context is used ("sentence"), and versions based on text context ("text") with or without our phrase improvement (" \pm phrase").

We evaluate using two standard metrics: accuracy ("Acc"), and macroaveraged F-score (" $\mathcal{F}_{\mathrm{M}}$ ").

\section{Results}

The results in Table 2 show that our system easily bests the off-the-shelf systems when it is given

\footnotetext{
${ }^{4}$ https://opennlp.apache.org/

${ }^{5} \mathrm{http}: / /$ alias-i.com/lingpipe
} 
the contextual information from the entire text; the difference is more stark for accuracy $(+0.085 \mathrm{ab}-$ solute), though consistent for $\mathcal{F}_{\mathrm{M}}(+0.041$ absolute). Stanford CoreNLP is the only competitive off-the-shelf system-the other two are far too conservative when encountering names they haven't seen before. LitNER is also clearly better than the Brown clusters it was trained on, particularly for $\mathcal{F}_{\mathrm{M}}$ (+0.120 absolute). With regards to different options for LitNER, we see a major benefit from considering all occurrences of the name in the texts rather than just the one we are testing on (Section 3.3), and a more modest benefit from using the information on parts of phrases taken from the Brown clustering (Section 3.4).

For the segmentation errors, we compared our corrected segmentations with the segmentation provided by the CRF-based Stanford CoreNLP system, our best competitor. Only 2 of the 15 were segmented correctly by Stanford CoreNLP. This potential 0.002 improvement is tiny compared to the 0.085 difference in accuracy between the two systems.

\section{Discussion}

Aspects of the method presented here could theoretically be applied to NER in other genres and other languages, but one important point we wish to make is that our approach clearly takes advantage of specific properties of (English) literature. The initial rule-based segmentation, for instance, depends on reliable capitalization of names, which is often not present in social media, or in most nonEuropean languages. We have found more subtle genre effects as well: for comparison, we applied the preliminary steps of our approach to another corpus of published texts which is of comparable (token) size to the Project Gutenberg corpus, namely the Gigaword newswire corpus (Graff and Cieri, 2003), and noted degraded performance for both segmentation and Brown clustering. With respect to the former, the obvious issue is considerably more complex proper nouns phrases such as governmental organizations and related titles. For the latter, there were several clusters in the top 10 (including the first one) which corresponded to LOCATION, while the first (fairly) clean PERSON cluster was the 15th largest; in general, individual people, organizations, and other groupings of people (e.g. by country of origin) were not well distinguished by Brown clustering in the Gigaword corpus, at least not with the same low number of clusters that worked well in the Project Gutenberg corpus.

Also less than promising is the potential for using text-level classification in other genres: whereas the average number of token occurrences of distinct name types within a single text in the Project Gutenberg corpus is 5.9, this number is just 1.6 for the much-shorter texts of the Gigaword corpus. Except in cases where it is possible to collapse texts into appropriately-sized groups where the use of a particular name is likely to be both common and consistent-an example might be a collection of texts written by a single author, which in social media such as Twitter seems to obey the classic one-sense-per-discourse rule (Gella et al., 2014) - it's not clear that this approach can be applied successfully in cases where texts are relatively short, which is a far more common situation. We also note that relying primarily on contextual classification while eschewing resources such as gazetteers makes much less sense outside the context of fiction; we would expect relatively few fictitious entities in most genres.

LitNER tags names into only two main classes, PERSON and LOCATION, plus a catch-all OTHER. This coarse-grained tag set reflects not only the practical limitations of the method, but also where we believe automatic methods have potential to provide useful information for literary analysis. The other clusters in Table 1 reflect word categories which are relatively closed-class and much less central to the fictional narratives as character and setting; we don't see a compelling case for tagging them. When these and non-entities are excluded from OTHER, what remains is eclectic, including names referring to small groups of people (e.g. families), animals, gods, ships, and titles of other works of literature.

\section{Conclusion}

In this paper, we have presented LitNER, an NER system targeted specifically at fiction. Our results show that a simple classifier, trained only with noisy examples derived in an unsupervised fashion, can easily beat a general-purpose supervised system, provided it has access to the full context of the text. Finally, we note that the NER tagging provided by LitNER has been integrated into the GutenTag tool (as of version 0.1.4). ${ }^{6}$

\footnotetext{
${ }^{6}$ See http://www.projectgutentag.org
} 


\section{References}

Apoorv Agarwal, Anup Kotalwa, and Owen Rambow. 2013. Automatic extraction of social networks from literary text: A case study on Alice in Wonderland. In The Proceedings of the 6th International Joint Conference on Natural Language Processign (IJCNLP'13).

Mariona Coll Ardanuy and Caroline Sporleder. 2015. Clustering of novels represented as social networks. Linguistic Issues in Language Technology, 12(4).

David Bamman, Ted Underwood, and Noah A. Smith. 2014. A Bayesian mixed effects model of literary character. In Proceedings of the 52st Annual Meeting of the Association for Computational Linguistics (ACL'14).

Julian Brooke, Adam Hammond, and Graeme Hirst. 2015. GutenTag: An NLP-driven tool for digital humanities research in the Project Gutenberg corpus. In Proceedings of the 4nd Workshop on Computational Literature for Literature (CLFL'15).

Peter F. Brown, Peter V. deSouza, Robert L. Mercer, Vincent J. Della Pietra, and Jenifer C. Lai. 1992. Class-based n-gram models of natural language. Computational Linguistics, 18(4):467-479.

Leon Derczynski, Sean Chester, and Kenneth S. Bgh. 2015. Tune your Brown clustering, please. In Proceedings of Recent Advances in Natural Language Processing (RANLP 15).

Micha Elsner, Eugene Charniak, and Mark Johnson. 2009. Structured generative models for unsupervised named-entity clustering. In Proceedings of the 2009 Annual Conference of the North American Chapter of the Association for Computational Linguistics: Human Language Technologies (NAACL '09).

David K. Elson, Nicholas Dames, and Kathleen R. McKeown. 2010. Extracting social networks from literary fiction. In Proceedings of the 48th Annual Meeting of the Association for Computational Linguistics (ACL'10).

Jenny Rose Finkel, Trond Grenager, and Christopher Manning. 2005. Incorporating non-local information into information extraction systems by gibbs sampling. In Proceedings of the 43rd Annual Meeting on Association for Computational Linguistics (ACL '05).

William A. Gale, Kenneth W. Church, and David Yarowsky. 1992. One sense per discourse. In Proceedings of the 4th DARPA Speech and Natural Language Workshop.

Spandana Gella, Paul Cook, and Timothy Baldwin. 2014. One sense per tweeter ... and other lexical semantic tales of twitter. In Proceedings of the 14th Conference of the European Chapter of the Association for Computational Linguistics.
David Graff and Christopher Cieri. 2003. English Gigaword. Linguistic Data Consortium.

Hua He, Denilson Barbosa, and Grzegorz Kondrak. 2013. Identification of speakers in novels. In Proceedings of the 51st Annual Meeting of the Association for Computational Linguistics (ACL '13).

Percy Liang. 2005. Semi-supervised learning for natural language. Master's thesis, MIT.

Mitchell P. Marcus, Beatrice Santorini, and Mary Ann Marcinkiewicz. 1993. Building a large annotated corpus of English: the Penn treebank. Computational Linguistics, 19(2):313-330.

Tara McIntosh, Lars Yencken, James R. Curran, and Timothy Baldwin. 2011. Relation guided bootstrapping of semantic lexicons. In Proceedings of the 49th Annual Meeting of the Association for Computational Linguistics: Human Language Technologies (ACL HLT 2011).

Scott. Miller, Jethran. Guinness, and Alex Zamanian. 2004. Name tagging with word clusters and discriminative training. In Proceedings of the 2004 Conference of the North American Chapter of the Association for Computational Linguistics: Human Language Technologies (NAACL HLT'13).

David Nadeau, Peter D. Turney, and Stan Matwin. 2006. Unsupervised named-entity recognition: Generating gazetteers and resolving ambiguity. In Proceedings of the 19th International Conference on Advances in Artificial Intelligence: Canadian Society for Computational Studies of Intelligence, AI'06.

F. Pedregosa, G. Varoquaux, A. Gramfort, V. Michel, B. Thirion, O. Grisel, M. Blondel, P. Prettenhofer, R. Weiss, V. Dubourg, J. Vanderplas, A. Passos, D. Cournapeau, M. Brucher, M. Perrot, and E. Duchesnay. 2011. Scikit-learn: Machine learning in Python. Journal of Machine Learning Research, 12:2825-2830.

Lev Ratinov and Dan Roth. 2009. Design challenges and misconceptions in named entity recognition. In Proceedings of the Thirteenth Conference on Computational Natural Language Learning (CoNLL '09).

Alan Ritter, Sam Clark, Mausam, and Oren Etzioni. 2011. Named entity recognition in tweets: An experimental study. In Proceedings of the 2011 Conference on Empirical Methods in Natural Language Processing (EMNLP 2011).

Michael Thelen and Ellen Riloff. 2002. A bootstrapping method for learning semantic lexicons using extraction pattern contexts. In Proceedings of the 2002 Conference on Empirical Methods in Natural Language Processing (EMNLP 2002).

Hardik Vala, David Jurgens, Andrew Piper, and Derek Ruths. 2015. Mr. Bennet, his coachman, and the Archbishop walk into a bar but only one of them gets 
recognized: On the difficulty of detecting characters in literary texts. In Proceedings of the 2015 Conference on Empirical Methods in Natural Language Processing (EMNLP'15).

Byron C. Wallace. 2012. Multiple narrative disentanglement: Unraveling Infinite Jest. In Proceedings of the 2012 Conference of the North American Chapter of the Association for Computational Linguistics: Human Language Technologies (NAACL-HLT'12). 